\title{
Conformal Invariance and Conserved Quantities of Mei Symmetry for Lagrange Systems
}

\author{
JiAN-LE CAI* \\ College of Science, Hangzhou Normal University, Hangzhou 310018, China
}

(Received February 2, 2009)

\begin{abstract}
Conformal invariance and conserved quantities of the Mei symmetry for the Lagrange systems under the infinitesimal transformation of groups are studied. Firstly, the definition of conformal invariance of the Mei symmetry is given, together with its criterion equations and determining equations. Then the systems' conserved quantities are obtained using the structure equation satisfied by the gauge function. Lastly, an example is taken to illustrate the application of the result.
\end{abstract}

PACS numbers: 02.20.Sv, 11.30.-j

\section{Introduction}

The symmetries and conserved quantities for a dynamical system play an important role in the fields of modern mathematics, mechanics and physics. The symmetry method is a modern approach for finding conserved quantities of dynamical systems. There are three main symmetry methods used to obtain the conserved quantities of dynamical systems: the Noether symmetry [1-6], the Lie symmetry [7-9] and the Mei symmetry [10-12], which lead to the three kinds of conserved quantities, i.e., the Noether conserved quantities, the Hojman conserved quantities and the Mei conserved quantities, respectively [13-16]. In Refs. [17-26] researchers have studied symmetries and conserved quantities for the Lagrange systems. The author of Ref. [12] has conducted comprehensive research to the above three kinds of main symmetries and associated conserved quantities for each type of restraint mechanical systems.

Conformal invariance is built on the scale invariance, translation invariance, rotational invariance and a variety of interactions. The method is of great theoretical significance and application value. Galiullin et al. [27] have studied conformal invariance, or conformal symmetry, of Birkhoff systems under special infinitesimal transformations. In Refs. [28-30] we have studied conformal invariance of general holonomic systems and Hamilton systems. The author of Ref. [31] has conducted conformal invariance of first-order differential equations. In this work, we study conformal invariance and conserved quantities of the Mei symmetry for Lagrange systems.

\section{The Mei symmetry of Lagrange systems}

Mei symmetry refers to the invariance of forms of system's equations of motion when their dynamical func-

\footnotetext{
* e-mail: caijianle@yahoo.com.cn
}

tions are transformed under infinitesimal transformations of groups. Consider a Lagrange system determined by the $n$ generalized coordinates $q_{s}(s=1, \ldots, n)$, and its differential equations of motion can be expressed as

$$
\begin{gathered}
\quad \frac{\mathrm{d}}{\mathrm{d} t} \frac{\partial L}{\partial \dot{q}_{s}}-\frac{\partial L}{\partial q_{s}}=0 \quad s=1, \ldots, n, \\
\text { or } \quad E_{s}(L)=0,
\end{gathered}
$$

where $q_{s}(s=1, \ldots, n)$ are generalized coordinates, $\dot{q}_{s}$ are generalized velocities, $L=L(t, \boldsymbol{q}, \dot{\boldsymbol{q}})$ is Lagrangian and

$$
E_{s}=\frac{\mathrm{d}}{\mathrm{d} t} \frac{\partial}{\partial \dot{q}_{s}}-\frac{\partial}{\partial q_{s}}
$$

are the Euler operators.

In general, let us assume that the system is nonsingular, i.e.:

$$
\operatorname{det}\left(\frac{\partial^{2} L}{\partial \dot{q}_{s} \partial \dot{q}_{k}}\right) \neq 0, \quad s, k=1, \ldots, n,
$$

then from Eq. (1) all the generalized accelerations can be calculated as

$$
\ddot{q}_{s}=\alpha_{s}(t, q, \dot{q}) \quad s=1, \ldots, n .
$$

Having in mind the symmetry inherent in Eq. (2), a single-parameter infinitesimal transformation with respect to time and coordinates can be introduced as

$$
t^{*}=t+\varepsilon \xi_{0}(t, \boldsymbol{q}, \dot{\boldsymbol{q}}), \quad q_{s}^{*}\left(t^{*}\right)=q_{s}(t)+\varepsilon \xi_{s}(t, \boldsymbol{q}, \dot{\boldsymbol{q}}),(6)
$$
where $\varepsilon$ is an infinitesimal transformation parameter, and $\xi_{0}, \xi_{s}$ are infinitesimal generators. Consider the infinitesimal generator vector

$$
X^{(0)}=\xi_{0} \frac{\partial}{\partial t}+\xi_{s} \frac{\partial}{\partial q_{s}},
$$

its first prolonged vector should be

$$
X^{(1)}=X^{(0)}+\left(\dot{\xi}_{s}-\dot{q}_{s} \dot{\xi}_{0}\right) \frac{\partial}{\partial \dot{q}_{s}} .
$$

Then suppose the Lagrangian $L$ is transformed into $L^{*}$ under the infinitesimal transformation in Eq. (6), we have 


$$
\begin{aligned}
L^{*} & =L\left(t^{*}, \boldsymbol{q}^{*}, \frac{\mathrm{d} \boldsymbol{q}^{*}}{\mathrm{~d} t^{*}}\right)=L(t, \boldsymbol{q}, \dot{\boldsymbol{q}})+\varepsilon X^{(1)}(L) \\
& +\mathrm{O}\left(\varepsilon^{2}\right),
\end{aligned}
$$

if we substitute $L$ with $L^{*}$, then the form of Eq. (2) remains unchanged, i.e.,

$$
E_{s}\left(L^{*}\right)=0
$$

we call such invariance as the Mei symmetry for the Lagrange systems.

Substituting Eq. (9) into Eq. (10) and ignoring high order small quantities, together with Eq. (2), we can get the determining equation of the Mei symmetry for the Lagrange system

$$
\left.E_{s}\left\{X^{(1)}(L)\right\}\right|_{E_{s}(L)=0}=0 .
$$

\section{Conformal invariance of the Mei symmetry of Lagrange systems}

Definition. For Lagrange equations in (2), if there exists a nonsingular matrix $M_{s}^{k}$ satisfying

$$
E_{s}\left\{X^{(1)}(L)\right\}=M_{s}^{k} E_{k}(L), \quad s, k=1, \ldots, n,
$$

then the Lagrange equations in (2) maintain conformal invariance of the Mei symmetry under the single-parameter infinitesimal transformations (6). Equation (12) is called the determining equations of conformal invariance for the Lagrange system in Eq. (2), and $M_{s}^{k}$ is called the conformal factor.

Theorem 1. For the Lagrangian $L=L(t, \boldsymbol{q}, \dot{\boldsymbol{q}})$, if the generators $\xi_{0}$ and $\xi_{s}$ of the infinitesimal transformations (6) satisfy

$$
X^{(1)}(L)=M L+C_{1} \dot{\varphi}+C_{2} \psi,
$$

then the Lagrange equations in Eq. (2) are called the conformal invariance of the Mei symmetry, where $C_{1}, C_{2}$ and $M$ are constants, $\varphi=\varphi(t, \boldsymbol{q}, \dot{\boldsymbol{q}}), \psi=\psi(t)$.

Proof. Since

$$
\begin{aligned}
& E_{s}(\dot{\varphi})=E_{s}\left(\frac{\partial \varphi}{\partial t}+\frac{\partial \varphi}{\partial q_{k}} \dot{q}_{k}+\frac{\partial \varphi}{\partial \dot{q}_{k}} \ddot{q}_{k}\right) \\
& =\frac{\mathrm{d}}{\mathrm{d} t} \frac{\partial \varphi}{\partial q_{s}}-\frac{\partial}{\partial q_{s}}\left(\frac{\partial \varphi}{\partial t}+\frac{\partial \varphi}{\partial q_{k}} \dot{q}_{k}+\frac{\partial \varphi}{\partial \dot{q}_{k}} \ddot{q}_{k}\right)=0 \\
& E_{s}(\psi)=\frac{\mathrm{d}}{\mathrm{d} t} \frac{\partial \psi}{\partial \dot{q}_{s}}-\frac{\partial \psi}{\partial q_{s}}=0
\end{aligned}
$$

from Eqs. (13)-(15) we can get

$$
\begin{gathered}
E_{s}\left\{X^{(1)}(L)\right\}=M E_{s}(L)+C_{1} E_{s}(\dot{\varphi})+C_{2} E_{s}(\psi) \\
=M E_{s}(L)=M_{s}^{k}\left\{E_{k}(L)\right\}, \quad s, k=1, \ldots, n,
\end{gathered}
$$

where $M_{s}^{k}=\delta_{s}^{k} M\left(\delta_{s}^{k}=\left\{\begin{array}{l}1, s=k \\ 0, s \neq k\end{array}\right)\right.$. Then the Lagrange equations in Eq. (2) are called the conformal invariance of the Mei, symmetry.

Theorem 2. If a Lagrange system maintains conformal invariance of the Mei symmetry, then it maintains the Mei symmetry, too.

Proof. Substituting Eq. (2) into Eq. (16), we can obtain the determining equations of the Mei symmetry for
Lagrange equations in Eq. (2) as below

$$
\begin{aligned}
& \left.E_{s}\left\{X^{(1)}(L)\right\}\right|_{E_{s}(L)=0} \\
& =\left.M_{s}^{k}\left\{E_{k}(L)\right\}\right|_{E_{k}(L)=0}=0 .
\end{aligned}
$$

\section{Conformal invariance and conserved quantity of the Mei symmetry}

Under certain conditions, the conformal invariance of the Mei symmetry for the Lagrange systems can also lead to conserved quantities.

Theorem 3. For the Lagrange system (2), if the generators $\xi_{0}$ and $\xi_{s}$ of the infinitesimal transformations (6) satisfy the determining Eqs. (12) of conformal invariance for the Mei symmetry, and there exists a gauge function $G=G(t, \boldsymbol{q}, \dot{\boldsymbol{q}})$ satisfying the following structure equation:

$$
X^{(1)}(L) \dot{\xi}_{0}+X^{(1)}\left\{X^{(1)}(L)\right\}+\dot{G}=0 .
$$

Then the conformal invariance of the Mei symmetry for the Lagrange system (2) leads to the following conserved quantity:

$$
\begin{aligned}
I= & X^{(1)}(L) \xi_{0}+\frac{\partial X^{(1)}(L)}{\partial \dot{q}_{s}}\left(\xi_{s}-\dot{q}_{s} \xi_{0}\right) \\
& +G=\text { const. }
\end{aligned}
$$

Proof. Using the differential Eqs. (2) of motion for the Lagrange systems and the determining Eqs. (12) for the conformal invariance of the Mei symmetry, we can obtain

$$
E_{s}\left\{X^{(1)}(L)\right\}=M_{s}^{k} E_{k}(L)=0,
$$

therefore

$$
\begin{aligned}
\frac{\mathrm{d} I}{\mathrm{~d} t} & =\left(\frac{\partial X^{(1)}(L)}{\partial t}+\frac{\partial X^{(1)}(L)}{\partial q_{s}} \dot{q}_{s}+\frac{\partial X^{(1)}(L)}{\partial \dot{q}_{s}} \ddot{q}_{s}\right) \xi_{0} \\
& +X^{(1)}(L) \dot{\xi}_{0}+\frac{\mathrm{d}}{\mathrm{d} t} \frac{\partial X^{(1)}(L)}{\partial \dot{q}_{s}}\left(\xi_{s}-\dot{q}_{s} \xi_{0}\right) \\
& +\frac{\partial X^{(1)}(L)}{\partial \dot{q}_{s}}\left(\dot{\xi}_{s}-\ddot{q}_{s} \xi_{0}-\dot{q}_{s} \dot{\xi}_{0}\right)-X^{(1)}(L) \dot{\xi}_{0} \\
& -\frac{\partial X^{(1)}(L)}{\partial t} \xi_{0}-\frac{\partial X^{(1)}(L)}{\partial q_{s}} \xi_{s}-\frac{\partial X^{(1)}(L)}{\partial \dot{q}_{s}}\left(\dot{\xi}_{s}-\dot{q}_{s} \dot{\xi}_{0}\right) \\
& =\left(\xi_{s}-\dot{q}_{s} \xi_{0}\right)\left(\frac{\mathrm{d}}{\mathrm{d} t} \frac{\partial X^{(1)}(L)}{\partial \dot{q}_{s}}-\frac{\partial X^{(1)}(L)}{\partial q_{s}}\right) \\
& =\left(\xi_{s}-\dot{q}_{s} \xi_{0}\right) E_{s}\left\{X^{(1)}(L)\right\}=0
\end{aligned}
$$

\section{An illustrative example}

For example, the differential equations of motion for a mechanic system with $n$ degrees of freedom are

$$
\ddot{q}_{s}+\frac{2}{t} \dot{q}_{s}+q_{s}^{5}=0, \quad s=1, \ldots, n .
$$

It can be transformed into the Lagrange system, as the 
corresponding Lagrangian is

$$
L=t^{2}\left[\frac{1}{2}\left(\dot{q}_{1}^{2}+\ldots+\dot{q}_{n}^{2}\right)-\frac{1}{6}\left(q_{1}^{6}+\ldots+q_{n}^{6}\right)\right] .
$$

Taking

$$
\xi_{0}=t, \quad \xi_{1}=-\frac{1}{2} q_{1}, \ldots, \xi_{n}=-\frac{1}{2} q_{n},
$$

we have

$$
\begin{gathered}
X^{(1)}(L)=-t^{2}\left[\frac{1}{2}\left(\dot{q}_{1}^{2}+\ldots+\dot{q}_{n}^{2}\right)\right. \\
\left.-\frac{1}{6}\left(q_{1}^{6}+\ldots+q_{n}^{6}\right)\right]=-L,
\end{gathered}
$$

therefore

$$
\begin{aligned}
& E_{s}\left\{X^{(1)}(L)\right\}=E_{s}(M L)=-\delta_{s}^{k} E_{k}(L)=0, \\
& \quad s, k=1, \ldots, n,
\end{aligned}
$$

which implies that the system maintains the Mei symmetry as well as conformal invariance of the Mei symmetry.

From (18), we can obtain

$$
\dot{G}=-X^{(1)}(L) \dot{\xi}_{0}-X^{(1)}\left\{X^{(1)}(L)\right\}=0,
$$

taking

$$
G=0
$$

the conserved quantity (19) gives

$$
\begin{aligned}
I & =\frac{1}{2} t^{3}\left(\dot{q}_{1}^{2}+\ldots+\dot{q}_{n}^{2}\right)+\frac{1}{2} t^{2}\left(q_{1} \dot{q}_{1}+\ldots+q_{n} \dot{q}_{n}\right) \\
& +\frac{1}{6} t^{3}\left(q_{1}^{6}+\ldots+q_{n}^{6}\right)=\text { const. }
\end{aligned}
$$

\section{Conclusions}

For a Lagrange system, under the single-parameter infinitesimal transformation, if the Lagrangian $L$ satisfies $X^{(1)}(L)=M L+C_{1} \dot{\varphi}+C_{2} \psi$ (where $\varphi=\varphi(t, \boldsymbol{q}, \dot{\boldsymbol{q}})$, $\psi=\psi(t), C_{1}, C_{2}$ and $M$ are constants), then the system maintains both conformal invariance of the Mei symmetry, and the Mei symmetry. The conformal invariance of the Mei symmetry can also lead to the corresponding conserved quantities due to certain circumstances.

\section{Acknowledgments}

Project supported by the National Natural Science Foundation of China (grant No. 10572021, 10772025).

\section{References}

[1] A.E. Noether, Nachr. Akad. Math. 2, 235 (1918).

[2] D.S. Djukić, B.D. Vujanović, Acta Mech. 23, 17 (1975).

[3] H.B. Wu, F.X. Mei, Acta Phys. Sin. 55, 3825 (2006).

[4] W.K. Ge, Acta Phys. Sin. 51, 1156 (2002).

[5] S.K. Luo, Chin. Sci. Bull. 36, 717 (1991).

[6] H.B. Zhang, Acta Phys. Sin. 50, 1837 (2001).

[7] F.X. Mei, G.H. Zheng, Acta Mech. Sin. 18, 414 (2002).

[8] F.X. Mei, Applications of Lie Groups and Lie Algebras to Constrained Mechanical Systems, Science Press, Beijing 1999.

[9] Y.Y. Zhao, F.X. Mei, Symmetries and Invariants of Mechanical Systems, Science Press, Beijing 1999.

[10] S.K. Luo, Acta Phys. Sin. 52, 2941 (2003).

[11] J.H. Fang, Commun. Theor. Phys. 40, 269 (2003).

[12] F.X. Mei, Symmetries and Conserved Quantities of Constrained Mechanical Systems, Beijing Institute of Technology Press, Beijing 2004.

[13] S.K. Luo, Acta Phys. Sin. 52, 1 (2003).

[14] Y. Zhang, Acta Phys. Sin. 54, 2980 (2005).

[15] L.Q. Jia, Y.Y. Zhang, S.W. Zheng, Acta Phys. Sin. 56, 0649 (2007).

[16] L.Q. Jia, S.W. Zheng, Y.Y. Zhang, Acta Phys. Sin. 56, 5575 (2007).

[17] Y. Zhang, F.X. Mei, Acta Phys. Sin. 53, 661 (2004).

[18] F.X. Mei, J. Beijing Inst. Tech. 25, 283 (2005).

[19] F.X. Mei, Acta Phys. Sin. 54, 2474 (2005).

[20] F.X. Mei, M. Shang, Acta Phys. Sin. 49, 1901 (2000).

[21] F.X. Mei, H.P. Zhu, J. Beijing Inst. Tech. 9, 11 (2000).

[22] R.C. Zhang, X.W. Chen, F.X. Mei, Chin. Phys. 9, 561 (2000).

[23] F.X. Mei, X.J. Xu, Chin. Phys. 14, 449 (2005).

[24] X.J. Xu, F.X. Mei, J. Beijing Inst. Tech. 24, 16 (2004).

[25] J.H. Fang, Y. Peng, Y.P. Liao, Acta Phys. Sin. 54, 496 (2005).

[26] Z.M. Lou, Acta Phys. Sin. 54, 1015 (2005).

[27] A.S. Galiullin, G.G. Gafarov, R.P. Malaishka, A.M. Khwan, Analytical Dynamics of Helmholtz, Birkhoff and Nambu Systems, UFN, Moscow 1997, p. 183.

[28] J.L. Cai, Chin. Phys. Lett. 25, 1523 (2008).

[29] J.L. Cai, F.X. Mei, Acta Phys. Sin. 57, 5369 (2008).

[30] J.L. Cai, S.K. Luo, F.X. Mei, Chin. Phys. B 17, 3170 (2008).

[31] G. He, F.X. Mei, Chin. Phys. B 17, 2764 (2008). 